\title{
Development of Predictive Responses in Theory of Mind Brain Regions
}

Hilary Richardson*, Rebecca Saxe

Department of Brain and Cognitive Sciences

Massachusetts Institute of Technology

43 Vassar Street, 46-4021

Cambridge, MA 02139

*Corresponding Author: hilary.richardson@childrens.harvard.edu 


\begin{abstract}
When we watch movies, we consider the characters' mental states in order to understand and predict the narrative. Recent work in fMRI uses movie-viewing paradigms to measure functional responses in brain regions recruited for such mental state reasoning (the Theory of Mind ("ToM") network). Here, two groups of young children (n=30 3-4yo, $n=26$ 6-7yo) viewed a short animated movie twice while undergoing fMRI. As children get older, ToM brain regions were recruited earlier in time during the second presentation of the movie. This "narrative anticipation" effect is specific: there was no such effect in a control network of brain regions that responds just as robustly to the movie (the "Pain Matrix"). These results complement prior studies in adults that suggest that ToM brain regions play a role not just in inferring, but in actively predicting, other people's thoughts and feelings, and provide novel evidence that as children get older, their ToM brain regions increasingly make such predictions.
\end{abstract}

\title{
Keywords:
}

Predictive Coding, Theory of Mind, fMRI, Cognitive Development, Anticipatory Responses 
Humans engage in sophisticated social cognitive reasoning: we reason about unobservable mental states - i.e., use our "Theory of Mind" (ToM; Gopnik \& Wellman, 1994) - proactively, in order to predict the actions and reactions of other people.

Indeed, fMRI studies of adults suggest that brain regions recruited for ToM reasoning (bilateral temporoparietal junction, precuneus, and prefrontal cortex; Carrington \& Bailey, 2009) not only infer, but actively predict, mental states. For example, ToM brain regions respond more when processing unexpected actions of an agent, given priors about her mental states (Dungan, Stepanovic, \& Young, 2016; Theriault \& Young, 2017; Heil et al., 2019), and use a person's current emotion to predict future emotional states (Thornton, Weaverdyck, \& Tamir, 2019). These findings are consistent with a predictive coding framework (Koster-Hale \& Saxe, 2013), in which neural responses reflect the generation of predictions about a stimulus and the recognition of differences between these predictions and the observed stimulus (Rao \& Ballard, 1999; Friston \& Kiebel, 2009; Clark, 2013; de Lange, Heilbron, \& Kok, 2018).

Here, we use a repeated movieviewing paradigm to measure development of predictive responses in ToM regions. During the second presentation of

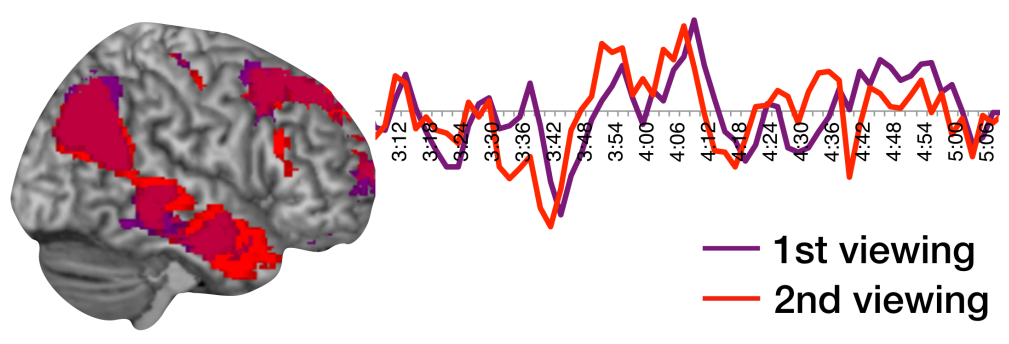
a movie, upcoming events are more predictable, so neural responses reflecting predictive processing should occur earlier during the second viewing (Figure 1). Initial evidence from adults supports this hypothesis: Baldassano et al. (2017) measured neural responses while adults listened to a narrative. Neural responses to the narrative

Figure 1. Visualization of Narrative Anticipation Effect. Visualization of ToM brain regions recruited for ToM reasoning during first (purple) and second (red) viewing of the movie "Partly Cloudy" (Reher \& Sohn, 2009), and average response timecourse from the ToM network per movie viewing (response magnitude on the y-axis, time in the movie on the $\mathrm{x}$-axis). Both visualizations show actual data from 6-7 year old participants in this study $(n=26)$. The "narrative anticipation" effect is visible in the timecourse data: the response in the ToM network is shifted earlier during the second presentation of a movie stimulus (red; relative to purple). See Supplementary Figure 1 for similar visualizations in younger children and in the Pain Matrix, and Supplementary Figure 3 for results of whole-brain random effects analyses per age group and movie viewing. 
shifted earlier in time in adults who had previously watched a movie version of the narrative, suggesting that these participants anticipated upcoming events during the second (audio) presentation. Intriguingly, the brain regions that showed predictive responses correspond to those canonically recruited for ToM reasoning (Baldassano et al., 2017).

The current study provides evidence for age-related change in these predictive responses in ToM brain regions in early childhood. Children's theory of mind changes dramatically between ages 3 and 7 years (e.g., Wellman \& Liu, 2004). However, children younger than six years of age are understudied with fMRI due to the demands of typical fMRI experiments. In the current study, two groups of young children (ages 3-4 and 6-7 years old) watched "Partly Cloudy" (Reher \& Sohn, 2009) twice while undergoing fMRI. This movie is short, engaging, and evokes responses in ToM brain regions, as well as a functionally distinct network of brain regions that respond to physical or bodily sensations (the "Pain Matrix"; Richardson et al., 2018). We measured the extent to which the response timecourse in ToM brain regions shifted earlier in time during the second viewing, indicating anticipation of the characters' mental states. We tested if anticipatory responses correlated with age and behavioral ToM reasoning, and conducted similar analyses in the Pain Matrix to determine if such anticipation is specific to ToM brain regions.

\section{Methods}

\section{Participants}

30 3.5-4 year olds $(\mathrm{M}(\mathrm{SD})=4(.39)$ years; 17 females, 2 left-handed/1 ambidextrous) and 26 6-7 year olds $(\mathrm{M}(\mathrm{SD})=6.5(.27) ; 14$ females, 6 left-handed/1 ambidextrous $)$ participated in the study. An additional 6 children were recruited to participate and excluded from all analyses for not completing the study. Children were recruited from the local community; 6-7 year old children were initially recruited for a longitudinal study. Parent/guardian consent and child assent was obtained prior to participation. Recruitment and experiment protocols were approved by the Committee on the Use of Humans as Experimental Subjects (COUHES) at the Massachusetts Institute of Technology.

The sample size was determined by the number of participants who completed two runs of a movie-viewing experiment included in two separate studies (one for 3-4 year olds (Richardson et 
al., 2018), one for 6 year olds (Richardson et al., in revisions)) that were designed and conducted to test hypotheses distinct from those tested here. Data from $3-4$ year old participants (first movie viewing only) were included in a previous publication (Richardson et al., 2018).

\section{Behavioral Battery}

All participants completed a Theory of Mind behavioral battery after the fMRI scan, listening to a story and answering 34 prediction $(n=20)$ and explanation $(n=14)$ questions about the beliefs, desires, and emotions of the characters, in the context of helping them find their snacks. This task taps a range of ToM concepts and asks children to reason about moral blameworthiness and second-order false-beliefs. In a prior study, performance on this task correlated with responses in ToM brain regions to social scenes during a naturalistic movie (Richardson et al., 2018). This task is publicly available (https://osf.io/G5ZPV/; DOI: 10.17605/OSF.IO/G5ZPV; ARK: c7605/osf.io/g5zpv).

\section{Behavioral Data Analysis}

Each child's Theory of Mind behavioral task was video recorded and coded offline. Theory of Mind behavioral performance was calculated as the number of questions answered correctly divided by the total number of questions asked. An additional five control items were asked to ensure that children were paying attention; after ensuring all children answered these questions correctly, these items were not further analyzed.

\section{fMRI Experiment}

Participants watched a silent version of "Partly Cloudy," (Reher \& Sohn, 2009) a 5.6-minute animated movie, twice. A short description of the plot can be found online (https://www.pixar.com/partly-cloudy\#partly-cloudy-1). This movie has previously been shown to drive neural responses in ToM brain regions in children (Richardson et al., 2018). The movie was preceded by 10 s of rest, and there was a short $(<1$ min) break between the two viewings during which time the experimenter briefly checked in with the participant. Participants were instructed to watch the movie and remain still. Children older than age five completed an additional fMRI experiment prior to viewing the movie, which involved listening to stories.

\section{fMRI Data Collection}


All participants practiced in a "mock scan" prior to the real fMRI scan. Mock scan sessions reduce participant motion and help young children become comfortable in the scanner environment.

During the mock and real scans, participants could hold a large, plush stuffed animal. Anecdotally, this stuffed animal helped children relax, reduced fidgeting with hands, and prevented children from touching their faces. Two other strategies employed to reduce participant motion were 1) packing the space between the child's head and the coil with soft foam pillows, and 2) having an experimenter remain by the child's foot, who tapped them on the leg if they started to move. This experimenter also ensured that participants were awake and attending to the movie. A second experimenter remained at the control console, and communicated with the children via a two-way microphone.

Whole-brain structural and functional MRI data were acquired on a 3-Tesla Siemens Tim Trio scanner located at the Athinoula A. Martinos Imaging Center at MIT. Children under age five years used one of two custom 32-channel phased-array head coils made for younger $(n=3$, $\mathrm{M}(\mathrm{SD})=3.91(.42)$ years) or older $(\mathrm{n}=27, \mathrm{M}(\mathrm{SD})=(4.05$ (.39) years) children (Keil et al., 2011); these children used the smallest coil that they fit in comfortably. Older children used the standard Siemens 32-channel head coil. T1-weighted structural images were collected in 176 interleaved sagittal slices with $1 \mathrm{~mm}$ isotropic voxels (GRAPPA parallel imaging, acceleration factor of 3; standard coil: FOV: 256mm; pediatric coils: FOV: 192mm). Functional data were collected with a gradient-echo EPI sequence sensitive to Blood Oxygen Level Dependent (BOLD) contrast in 32 interleaved near-axial slices with $3 \mathrm{~mm}$ isotropic voxels and a $10 \%$ slice gap, aligned with the anterior/posterior commissure, and covering the whole brain (EPI factor: 64; TR: 2s, TE: $30 \mathrm{ms,}$ flip angle: $90^{\circ}$ ). All functional data were subsequently upsampled in normalized space to $2 \mathrm{~mm}$ isotropic voxels. Prospective acquisition correction was used to adjust the positions of the gradients based on the participant's head motion one TR back (Thesen, Heid, Mueller, \& Schad, 2000). 168 volumes were acquired in each run; the two movie viewings were collected across two runs. Four dummy scans were collected to allow for steady-state magnetization.

FMRI Analysis 
FMRI data were analyzed in SPM8 (http://www.fil.ion.ucl.ac.uk/spm) and custom software written in Matlab (MathWorks, Natick, MA) and R (https://www.r-project.org/). Functional images were registered to the first image of the run; that image was registered to each participant's anatomical image, and each participant's anatomical image was normalized to the Montreal Neurological Institute (MNI) template. This enabled us to use group regions of interest (ROIs) and hypothesis spaces previously created in adult datasets and used to study responses in children. Previous research has suggested that anatomical differences between children as young as seven years are small relative to the resolution of fMRI data, which supports usage of a common space between adults and children of this age (for similar procedures with children under age seven, see Cantlon \& Li, 2013; Richardson et al., 2018); for methodological considerations, see Burgund et al., 2002). Registration of each individual's brain to the MNI template was visually inspected, including checking the match of the cortical envelope and internal features like the AC-PC and major sulci. All data were smoothed using a Gaussian filter (5mm kernel), and underwent SPM's image scaling.

Artifact timepoints were identified via the ART toolbox (https://www.nitrc.org/projects/artifact_detect/; Whitfield-Gabrieli, Nieto-Castanon, \& Ghosh, 2011) as timepoints for which there was 1) more than $2 \mathrm{~mm}$ composite motion relative to the previous timepoint or 2) a fluctuation in global signal that exceeded a threshold of three standard deviations from the mean global signal. Participants would have been excluded if one-third or more of the timepoints collected were identified as artifact timepoints; zero participants met this criterion. Number of artifact timepoints positively correlated with mean translation $(r(54)=.38$, $\mathrm{p}=.004)$. Overall, amount of motion was minimal (mean translation in non-artifact timepoints: $1^{\text {st }}$ viewing (V1): Young children: $\mathrm{M}(\mathrm{SD})=.06(.03) \mathrm{mm}$; older children: $\mathrm{M}(\mathrm{SD})=.07(.03) \mathrm{mm} ; 2^{\text {nd }}$ viewing (V2): Young children: $\mathrm{M}(\mathrm{SD})=.06(.03) \mathrm{mm}$; older children: $\mathrm{M}(\mathrm{SD})=.08(.04) \mathrm{mm})$. Average number of artifact timepoints did not differ between old and young children in either viewing (V1: Young children: $\mathrm{M}(\mathrm{SD})=9.3(10.8)$; Older children: $\mathrm{M}(\mathrm{SD})=13.4(15.4)$; V2: Young children: $\mathrm{M}(\mathrm{SD})=13.3(13.3)$; Older children: $\mathrm{M}(\mathrm{SD})=13.4(13.3)$; ps $>.2)$, and average number of artifact timepoints was not correlated with age (spearman correlation: $r_{s}(54)=.13, \mathrm{p}=.32$ ) or ToM behavioral performance (kendall tau correlation: $r_{k}(54)=-.06, \mathrm{p}=.5$ ). Number of artifact timepoints did not differ between movie viewings in either age group (Young children: $t(55.6)=-$ 
1.3, $\mathrm{p}=.20$; Older children: $\mathrm{t}(49)=-.01, \mathrm{p}=.99)$. See Supplementary Figure 2 for a visualization of participant motion by movie viewing and age group. Despite amount of motion being uncorrelated with age, and therefore likely not driving developmental effects within the child sample, we included number of motion artifact timepoints as a covariate in all analyses. Because this measure was not normally distributed, spearman correlations were used when including amount of motion as a covariate in partial correlations.

For the purpose of creating Figure 1, whole-brain random effects analyses were used to show the main contrast of interest (ToM events > Pain events; events described in Richardson et al., 2018 and https://openneuro.org/datasets/ds000228) per age group and movie viewing. These analyses were corrected for multiple comparisons by estimating the false-positive rate via 5,000 Monte Carlo permutations using the SnPM toolbox for SPM5 (http://www.fil.ion.ucl.ac.uk/spm/software/spm5/; $\mathrm{p}<.05$ ). See Supplementary Figure 3 for visualization of results for each age group and movie viewing.

For the main analyses, region of interest (ROI) analyses were conducted using ToM and "Pain Matrix" group ROIs. The "Pain Matrix" was selected as a control network for the current analyses, as previous work has found that, like the ToM network, responses in these regions are driven by this movie (Richardson et al., 2018). These ROIs were previously created in an independent group of adults, based on responses to the "Partly Cloudy" movie $(n=20)$. The ROI creation procedure is described in a prior publication (Richardson et al., 2018), and the ROIs are publicly available for download (https://openneuro.org/datasets/ds000228). In two prior studies, the magnitude of response in these ToM ROIs to social scenes in naturalistic movies correlated with behavioral measures of social reasoning in children, even when controlling for age (Richardson et al., 2018; Richardson, 2018).

All timecourse analyses were conducted by extracting the preprocessed timecourse from each voxel per group ROI (per movie viewing). We applied nearest neighbor interpolation over artifact timepoints, and regressed out two kinds of nuisance covariates to reduce the influence of motion artifacts: 1) motion artifact timepoints, and 2) five principle component analysis (PCA)based noise regressors generated using CompCor within individual subject white matter masks 
(Behzadi, Restom, Liau, \& Liu, 2007). White matter masks were eroded by two voxels in each direction, in order to avoid partial voluming with cortex. CompCor regressors were defined using scrubbed data (e.g. artifact timepoints were identified and interpolated over prior to running CompCor). The residual timecourses were then high-pass filtered with a cutoff of 100 seconds. Timecourses from all voxels within an ROI were averaged, creating one timecourse per group ROI, and artifact timepoints were subsequently excluded (NaNed). ROI timecourses within each network were averaged, creating one timecourse per network, per movie viewing and participant.

We then calculated the correlation between each participant's timecourses during the first and second viewings for the ToM and pain network, separately, in two temporal shifting schemes. In the "no shift" scheme, we calculated the correlation between timepoints 1 to 168 in the first and second viewings. In the "anticipation" scheme, we calculated the correlation between timepoints 2 to 168 in the first viewing and timepoints 1 to 167 in the second viewing. Correlation values were z-scored and compared directly by calculating the "anticipation" - "no shift" correlation difference, in order to determine if the timecourses were more correlated under the "anticipation" scheme.

In each network and age group, we used t-tests to determine if the "anticipation" - "no shift" difference score was significantly positive, suggesting that the brain regions in that network and age group anticipated events of the movie during the second viewing. We additionally used ttests to directly compare the size of this 'anticipation effect' across age groups, and used partial correlation tests to determine if the anticipation effect increased with age and a behavioral measure of theory of mind reasoning, controlling for participant motion. Finally, we used a linear mixed-effect model fit by maximum likelihood to test for main effects of age, network, and motion, and for an age $\mathrm{x}$ network interaction, in order to test the specificity of the effect (subject identifier was included as a random effect). The data used for analyses are available for download ([link forthcoming]).

\section{Results}

Behavioral Results 
All children completed a Theory of Mind behavioral battery after the scan, which involved listening to a story and answering prediction and explanation questions that require reasoning about the mental states of the characters (see Methods and https://osf.io/G5ZPV/). ToM performance correlated with age $\left(r_{s}(53)=.81, \mathrm{p}=1.2 \times 10^{-13}\right)$, and older children performed significantly better than younger children $\left(\mathrm{t}(43.4)=12.4, \mathrm{p}=8.2 \times 10^{-16}\right)$; see Supplementary Figure $4 a$.

\section{fMRI Results}

As expected given the identical movie stimulus across viewings, the response timecourses were highly correlated across the two viewings in both age groups and cortical networks ("no shift" correlation: ToM: Young: $\mathrm{M}(\mathrm{SE}) r=.11(.02) ; \mathrm{t}(29)=4.8, \mathrm{p}=4.8 \times 10^{-5}$; Older: $\mathrm{M}(\mathrm{SE}) r=.13(.02)$; $\mathrm{t}(25)=5.9, \mathrm{p}=4.2 \times 10^{-6}$; Pain: Young: $\mathrm{M}(\mathrm{SE}) r=.19(.03) ; \mathrm{t}(29)=5.9, \mathrm{p}=2.0 \times 10^{-6}$; Older: $\mathrm{M}(\mathrm{SE})$ $r=.25(.03) ; \mathrm{t}(25)=9.4, \mathrm{p}=1.2 \times 10^{-9}$; see Supplementary Figure 1. The "no shift" correlation between the two timecourses was uncorrelated with age in both networks (ToM: $r(53)=.14$, $\mathrm{p}=.31$; Pain: $r(53)=.13, \mathrm{p}=.35$; partial Pearson correlations including motion as a covariate), and did not differ between young and older children (ToM: $\mathrm{t}(53.6)=.78, \mathrm{p}=.22$; Pain: $\mathrm{t}(53.5)=1.5$, $\mathrm{p}=.07$; one-tailed t-tests).

In theory, temporally shifting one timecourse such that the two timecourses are no longer temporally aligned should reduce the correlation between them. By contrast, if a cortical network generates predictive responses to a familiar narrative, then shifting its response timecourse during the second viewing to be temporally earlier might increase the correlation between the two timecourses. We measured the anticipation effect as the "anticipation" - "no shift" correlation difference in each network, and tested for main effects of age group (3-4yo vs. 6-7yo) and network (ToM vs. Pain), as well as an age group x network interaction, controlling for motion. There was a significant age group $\mathrm{x}$ network interaction, such that the anticipation effect in the ToM network was larger in older children $(\mathrm{b}=.85, \mathrm{t}=2.3, \mathrm{p}=.03)$. The main effects of network $(\mathrm{b}=-.26, \mathrm{t}=-1, \mathrm{p}=.31)$, age group $(\mathrm{b}=-.22, \mathrm{t}=-.84, \mathrm{p}=.40)$, and motion $(\mathrm{b}=.05, \mathrm{t}=.55$, $\mathrm{p}=.58$ ) were not significant. Using age as a continuous variable yielded the same pattern of results, but the age $\mathrm{x}$ network interaction was marginal $(\mathrm{b}=.34, \mathrm{t}=1.8, \mathrm{p}=.08$; other $\mathrm{bs}<.15$, $\mathrm{ps}>.4$; see Figure 2a and Supplementary Figure 5). 
In 3-4 year olds, temporally misaligning the ToM timecourses from the first and second viewings reduced the correlation between them (anticipation effect $\mathrm{M}(\mathrm{SE})=-.03(.02)$; one-tailed t-test against zero: $\mathrm{t}(29)=-1.8, \mathrm{p}=.04)$. By contrast, in 6-7 year olds, timecourses in the ToM network were marginally more correlated when misaligned into the anticipation scheme (anticipation effect $\mathrm{M}(\mathrm{SE})=.025(.02)$, one-tailed t-test against zero: $\mathrm{t}(25)=1.54, \mathrm{p}=.07)$. The anticipation effect in the ToM network was significantly larger in older children than in younger children (two-tailed t-test: $\mathrm{t}(53)=2.3, \mathrm{p}=.02$ ). Across all children, the anticipation effect ("anticipation" "no shift" difference) in ToM brain regions positively correlated with age $(r(53)=.28, \mathrm{p}=.04$; partial Pearson correlation including motion as a covariate), but not with ToM behavior $(\mathbf{T}(53)=.18, \mathrm{p}=.2$; partial Kendall correlation including motion as a covariate); see Supplementary Figure 4b).
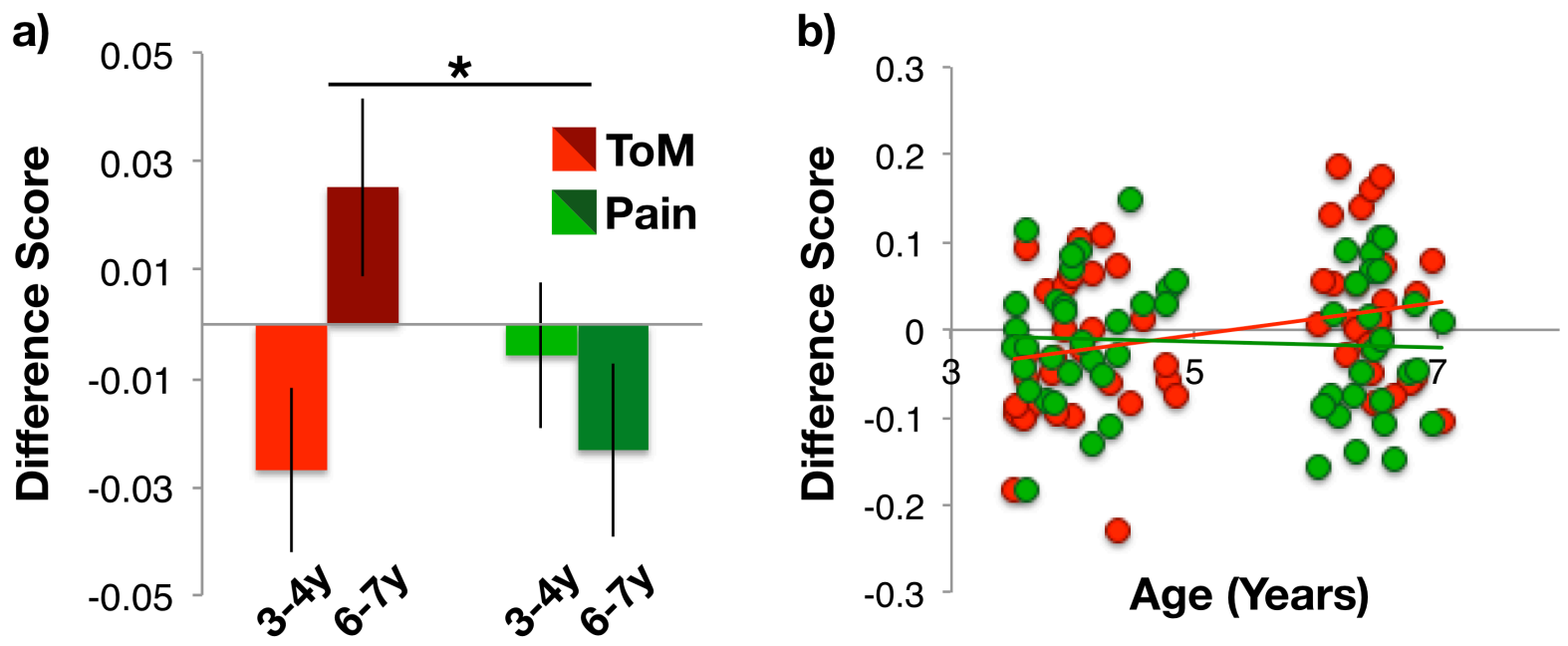

Figure 2. FMRI Results a) Bar plot shows difference score on the y-axis by age group (3-4 year olds in light colors, 6-7 year olds in dark colors) and network (ToM network in reds, Pain Matrix in greens). The difference score is the z-scored correlation between the response timecourses (within each network) during the first and second viewings under the "anticipation" scheme (i.e., timepoints 2:167 during the first viewing to timepoints 1:168 during the second viewing), minus the z-scored correlation between the same response timecourses under the "no shift" scheme (i.e., timepoints 1:168 during the first and second viewings). A positive difference score indicates that the timecourses were more correlated when temporally earlier timepoints during the second viewing were aligned with later timepoints in the first viewing. The asterisk indicates a significant age-group $\mathrm{x}$ network interaction $(\mathrm{p}<.05)$ such that the anticipation effect in the ToM network was significantly larger in older children. b) Scatterplot shows the same difference score (y-axis) by age as a continuous variable (x-axis). The narrative anticipation effect increases with 
age in the ToM network (controlling for motion: $r(53)=.28, \mathrm{p}=.04$ ), but not the Pain Matrix (controlling for motion: $r(53)=-.08, \mathrm{p}=.56$ ).

The anticipation effect in the Pain Matrix was not significantly positive in older (anticipation effect $\mathrm{M}(\mathrm{SE}) r=-.02(.02)$, one-tailed t-test: $\mathrm{t}(25)=-1.4, \mathrm{p}=.9)$ or younger (M(SE) $r=-.01(.01)$, onetailed t-test: $\mathrm{t}(29)=-.43, \mathrm{p}=.7)$ children, and did not change with age $(r(53)=-.08, \mathrm{p}=.56$, includes motion as covariate; two-tailed t-test comparing age groups: $\mathrm{t}(51)=-.84, \mathrm{p}=.41)$. The anticipation effect in the ToM network was significantly more correlated with age than the anticipation effect in the Pain Matrix (one-tailed r-test: $\mathrm{z}=1.9, \mathrm{p}=.03$ ).

Post-hoc exploratory analyses confirmed that the anticipation effect was most pronounced at a time lag of two seconds (Supplementary Figure 5), and that the focus on ToM brain regions did not result in missing similar anticipation effects elsewhere in the brain (Supplementary Figure 6, Supplementary Figure 7). The same pattern of results was obtained in post-hoc analyses of the response pattern, rather than univariate response magnitude (Supplementary Figure 8). See Supplementary Materials for exploratory analyses of repetition suppression in each network across the two viewings (Supplementary Figure 9) and of hippocampal responses at event boundaries (Supplementary Figure 10).

\section{Discussion}

Young children watched a short animated film twice while undergoing fMRI scanning. Between ages three and seven years, we observed increasing predictive neural responses to the second viewing. Thus using a repeated naturalistic movie-viewing paradigm allowed us to measure developmental change in neural predictive processing in very young children. This kind of paradigm is promising for measuring continued development of predictive responses in childhood, and for understanding the relationship between the development of neural predictive responses and conceptual knowledge in ToM.

In adults, prior knowledge of a narrative leads to earlier activation in a group of brain regions, including temporo-parietal junction, precuneus and medial prefrontal cortex (Baldassano et al. 2017). In the current data, narrative anticipation effects increased with age in a similar set of regions (here called Theory of Mind regions), and not in a control network that is similarly 
driven by viewing the movie (here called the Pain Matrix). What cognitive process underlies the anticipation effect in these regions? There are multiple possibilities that cannot be distinguished by the current data.

We suggest that anticipatory activity is specifically related to predicting and understanding the characters' mental states. In most naturalistic human-created narratives, characters' desires, expectations and emotions drive their actions and the narrative. Anticipation effects are observed in brain regions that are most active when the stimulus evokes consideration of characters' mental states (e.g., Carrington \& Bailey, 2009), and the magnitude and selectivity of activity in these brain regions have been related to children's theory of mind reasoning (Richardson et al., 2018).

However, an alternative is that these brain regions are involved more generally in representing events at long temporal scales, independent of event content (Baldassano et al., 2017; Jacoby \& Fedorenko, 2018). Furthermore, anticipation effects measured over the whole timecourse of movie viewing could reflect looking forward to as yet unseen events, or faster comprehension of ongoing events, or both; relatedly, anticipation effects could reflect earlier predictive processing, or reduced prediction error, or both. To distinguish between these hypotheses, it may be helpful to formalize these alternatives in computational terms, to use stimuli in which content and temporal structure are un-confounded, and to collect more data per participant.

Thus, this study motivates several questions for future research. In particular, what are the cognitive and behavioral consequences of more neural anticipation of narratives? We did not find a correlation with our measure of ToM behavior in the current sample; future experiments could test other behavioral measures of narrative comprehension and mentalistic prediction. Naturalistic movie-viewing paradigms are a promising approach for addressing these questions, and for understanding how the developing brain makes predictions about minds.

\section{Acknowledgements}

We thank the Athinoula A. Martinos Imaging Center at the McGovern Institute for Brain Research at MIT, and gratefully acknowledge support by a NSF Graduate Research Fellowship 
(\#1122374 to HR), Whitaker Health Sciences Fund Fellowship (HR), NSF Career award (\#095518 to RS), and the David and Lucile Packard Foundation (\#2008-333024 to RS). 


\section{References}

Baldassano, C., Chen, J., Zadbood, A., Pillow, J. W., Hasson, U., \& Norman, K. A. (2017). Discovering event structure in continuous narrative perception and memory. Neuron, 95(3), 709-721.

Behzadi, Y., Restom, K., Liau, J., \& Liu, T. T. (2007). A component based noise correction method (CompCor) for BOLD and perfusion based fMRI. NeuroImage, 37(1), 90-101.

Burgund, E. D., Kang, H. C., Kelly, J. E., Buckner, R. L., Snyder, A. Z., Petersen, S. E., \& Schlaggar, B. L. (2002). The Feasibility of a Common Stereotactic Space for Children and Adults in fMRI Studies of Development. NeuroImage, 17(1), 184-200. http://doi.org/10.1006/nimg.2002.1174

Cantlon, J. F., \& Li, R. (2013). Neural activity during natural viewing of Sesame Street statistically predicts test scores in early childhood. PLoS Biology, 11(1), e1001462.

Carrington, S. J., \& Bailey, A. J. (2009). Are there theory of mind regions in the brain? A review of the neuroimaging literature. Human Brain Mapping, 30(8), 2313-2335. http://doi.org/10.1002/hbm.20671

Clark, A. (2013). Whatever next? Predictive brains, situated agents, and the future of cognitive science. Behavioral and Brain Sciences, 36(3), 181-204.

de Lange, F. P., Heilbron, M., \& Kok, P. (2018). How do expectations shape perception? Trends in Cognitive Sciences.

Dungan, J. A., Stepanovic, M., \& Young, L. (2016). Theory of mind for processing unexpected events across contexts. Social Cognitive and Affective Neuroscience, 11(8), 1183-1192.

Friston, K., \& Kiebel, S. (2009). Predictive coding under the free-energy principle. Phil. Trans. R. Soc. B, 364(1521), 1211-1221.

Gopnik, A., \& Wellman, H. M. (1994). The theory theory. Mapping the Mind: Domain Specificity in Cognition and Culture, 257.

Heil, L., Colizoli, O., Hartstra, E., Kwisthout, J., van Pelt, S., van Rooij, I., \& Bekkering, H. (2019). Processing of Prediction Errors in Mentalizing Areas. Journal of Cognitive Neuroscience, 1-13.

Jacoby, N., \& Fedorenko, E. (2018). Discourse-level comprehension engages medial frontal Theory of Mind brain regions even for expository texts. Language, Cognition and Neuroscience, 1-17.

Keil, B., Alagappan, V., Mareyam, A., McNab, J. A., Fujimoto, K., Tountcheva, V., et al. (2011). Size-optimized 32-channel brain arrays for 3 T pediatric imaging. Magnetic Resonance in Medicine, 66(6), 1777-1787.

Koster-Hale, J. \& Saxe, R. (2013). Theory of Mind: A Neural Prediction Problem. Neuron, 79(5), 836-848. http://doi.org/10.1016/j.neuron.2013.08.020

Rao, R. P., \& Ballard, D. H. (1999). Predictive coding in the visual cortex: a functional interpretation of some extra-classical receptive-field effects. Nature Publishing Group, 2(1), 79.

Reher, K., \& Sohn, P. (2009). Partly Cloudy [Motion Picture]. USA: Pixar Animation Studios and Walt Disney Pictures.

Richardson, H. (2018). Development of Brain Networks for Social Functions: Confirmatory Analyses in a Large Open Source Dataset. Developmental Cognitive Neuroscience.

Richardson, H., Lisandrelli, G., Riobueno-Naylor, A., \& Saxe, R. (2018). Development of the social brain from age three to twelve years. Nature Communications, 9(1), 1027.

Theriault, J., \& Young, L. (2017). Social Prediction in the Theory of Mind Network. PsyArxiv. 
Thesen, S., Heid, O., Mueller, E., \& Schad, L. R. (2000). Prospective acquisition correction for head motion with image-based tracking for real-time fMRI. Magnetic Resonance in Medicine, 44(3), 457-465.

Thornton, M. A., Weaverdyck, M. E., \& Tamir, D. I. (2019). The social brain automatically predicts others' future mental states. Journal of Neuroscience, 39(1), 140-148.

Wellman, H. M., \& Liu, D. (2004). Scaling of theory-of-mind tasks. Child Development, 75(2), 523-541.

Whitfield-Gabrieli, S., Nieto-Castanon, A., \& Ghosh, S. (2011). Artifact Detection Tools (ART). Cambridge, MA. Release Version, 7(19), 11. 